\title{
Reason for Narrative
}

National Cancer Institute

\section{Source}

National Cancer Institute. Reason for Narrative. NCI Thesaurus. Code C99097.

The explanation why a summary was necessary for a clinical study. 